\title{
Prescrição de exames adicionais para realização de colonoscopia com sedação
}

Margarida Vaz Pinto, ${ }^{1}$ Paula Ponte, ${ }^{1}$ António Luz Pereira ${ }^{2}$

\section{RESUMO}

No contexto atual dos recursos em saúde, a procura de eficiência é um imperativo. Este é um dos princípios essenciais da medicina geral e familiar.

Objetivos: Caracterizar os procedimentos relativos a meios complementares de diagnóstico (MCDT) das instituições convencionadas com a ARS Norte para a realização de colonoscopia com sedação.

Metodologia: Durante o mês de março foram contactadas por telefone instituições convencionadas com a ARS Norte, que realizam colonoscopia sob sedação, na área de influência do ACeS Porto Ocidental. Foram colocadas questões relativas aos exames pedidos para realização de colonoscopia com sedação, prazo em que são válidos e procedimentos de acordo com faixa etária e comorbilidades.

Resultados: Vinte e duas instituições foram contactadas, tendo sido obtidas 19 respostas - taxa de resposta de 86\%; $100 \%$ das instituições pede estudo da coagulação, $95 \%$ hemograma com plaquetas; $58 \%$ função renal; $53 \%$ glicose; $42 \%$ ionograma; e $100 \%$ ECG. Relativamente ao ECG e a procedimentos específicos na presença de comorbilidades verificou-se que não existiam critérios uniformes. O prazo em que os exames são válidos variou entre três e seis meses. O custo total em MCDT variou entre um valor mínimo de 10,38 euros e um valor máximo de 41,66 euros. Considerando o número de colonoscopias realizadas no ano de 2015 no ACeS Porto Ocidental (3.072), estima-se que o custo destes exames possa variar entre $€ 31.887,36$ e $€ 127.979,52$, ou seja, a diferença de procedimentos envolve um valor estimado de $€ 96.092,16$.

Discussão: Verificaram-se diferenças no tipo de atendimento prestado, na clareza da informação fornecida, no conhecimento relativo aos exames complementares de diagnóstico e nos critérios que justificam o pedido de ECG.

Conclusões: Ficou clara a necessidade de elaborar um documento de consenso que permita diminuir a variabilidade destes procedimentos, evitar procedimentos/gastos desnecessários, garantindo a segurança e as boas práticas relativas a estes procedimentos.

Palavras-chave: Colonoscopia; Convencionados; Eficiência; Procedimentos associados.

\section{INTRODUÇÃO}

$\Lambda$ colonoscopia tem vindo a assumir uma importância crescente na atividade clínica diária pela sua potencialidade diagnóstica e terapêutica. Constitui o exame de primeira linha nos doentes que apresentam sinais e/ou sintomas sugestivos da existência de patologia do cólon ou do reto, tornando esta técnica fundamental no âmbito da patologia do cólon e do reto. ${ }^{1}$ Sendo um exame frequentemente acompanhado e condicionado pela dor,

1. Médica Interna de Medicina Geral e Familiar. USF Prelada 2. Médico Assistente de Medicina Geral e Familiar. USF Prelada a realização com sedação/anestesia deve estar disponível. Sabe-se que grande parte das colonoscopias são efetuadas sob sedação/anestesia e que, dependendo da farmacocinética e farmacodinâmica do fármaco utilizado, do perfil fisiológico do utente e da experiência do profissional, a sedação pode ser díficil de estabilizar e evoluir para anestesia. ${ }^{1}$

O rastreio oportunístico do cancro do cólon e reto é, em nosso entender, um verdadeiro desafio às premissas que constituem a integração funcional, uma vez que devem ser garantidos serviços prestados pelos vários profissionais, em vários locais ou organizações ao longo do tempo, de acordo com as necessidades espe- 
cíficas de cada utente, segundo um determinado nível de conhecimento e tecnologia disponível. ${ }^{2}$ Isto significa uma prestação de cuidados de saúde com valor para o utente, baseada na coordenação, comunicação e cooperação efetiva das funções e atividades básicas desenvolvidas nas unidades operacionais do sistema de produção. Só assim se garantirão cuidados de saúde mais custo-efetivos com qualidade. ${ }^{3}$

Os médicos de família são chamados diariamente a encontrar respostas adequadas para problemas específicos e necessidades específicas de saúde. Assim, dever-se-á procurar integrar conhecimento atualizado, homogeneizando as atuações com o objetivo de atingir resultados em termos de ganhos em saúde, com o menor custo possível, sem desperdícios ou gastos desnecessários, reduzindo desigualdades inaceitáveis em saúde. Este conjunto de práticas e atitudes permitem uma melhoria contínua da qualidade dos processos assistenciais e de intervenção em saúde, promovendo a motivação e satisfação dos profissionais, a capacitação, autonomia e satisfação dos utentes. ${ }^{4}$ Deste modo, devem ser identificadas todas as áreas em que as práticas efetivamente não se traduzem em melhores cuidados para os utentes. Essa identificação permitirá uma correção destas práticas com verdadeiros ganhos em eficiência.

Na nossa prática clínica somos confrontados com a necessidade de prescrição de exames adicionais para a realização de colonoscopia com sedação, sem qualquer suporte pelo clausulado que aprovou as convenções na área da endoscopia gastroenterológica.

Tendo observado que utentes com patologias e idade semelhantes solicitam diferentes protocolos para realização de endoscopia digestiva baixa com sedação, de acordo com o convencionado que escolheram, surgiu a necessidade de analisar os procedimentos das instituições convencionadas que servem a nossa comunidade.

\section{OBJETIVO}

Caracterizar os procedimentos das instituições convencionadas com a ARS Norte da nossa área de atuação para a realização de colonoscopia com sedação.

\section{MÉTODO}

Durante o mês de março de 2016 foram contactadas por telefone instituições que realizam colonoscopias sob sedação, convencionadas com a ARS Norte, tendo sido excluídas todas as que se encontravam fora da área geográfica usada pelos nossos utentes. A seleção foi feita através da listagem oficial disponível no site da ARS Norte obtida em 15/02/2016. Foram colocadas as seguintes questões: quais as análises e exames pedidos; qual o prazo em que os meios complementares de diagnóstico (MCDT) são considerados válidos; a distinção de procedimentos de acordo com faixa etária e comorbilidades. Os dados recolhidos foram analisados em Excel.

\section{RESULTADOS}

De uma lista de 22 instituições contactadas, duas já não realizam colonoscopia e noutra o número não se encontrava atribuído. Foram, portanto, obtidas respostas de um total de 19 instituições.

A totalidade das instituições requeria estudo da coagulação (INR), 95\% hemograma com plaquetas; $58 \%$ função renal; $53 \%$ glicose; e $42 \%$ ionograma. Relativamente à necessidade de ECG, $26 \%$ das unidades requeriam ECG independentemente da idade, enquanto todas as restantes o faziam de acordo com a idade do utente. Em 16\% das instituições, o ECG era requerido se a idade estivesse acima de 40 anos; enquanto outros $16 \%$ o faziam apenas quando a idade fosse superior a 45 anos. Em $32 \%$ das instituições e perante uma idade superior a 50 anos era requerido o ECG; num caso (6\%) se a idade fosse superior a 55 anos. Num outro caso era feita a distinção da idade de acordo com o género: mulheres com mais de 50 anos e homens com mais de 65 anos. A existência de patologia cardíaca, independentemente da idade, era razão para a necessidade de ECG em $11 \%$ das instituições. Verificou-se a existência de procedimentos diferentes relativamente ao pedido de ECG dentro do mesmo grupo de saúde, sendo que a idade requerida era superior a 45 anos num local e 50 anos noutro. Os dados obtidos encontram-se resumidos nos Quadros I e II.

Quanto ao prazo dos MCDT, 17 instituições referiram seis meses, outra quatro meses e ainda outra três meses. A maioria das instituições não apresentava procedimentos específicos de acordo com a presença de comorbilidades. Uma instituição referia necessidade de estudo detalhado das comorbilidades; uma, quando 


\begin{tabular}{l}
\hline \multicolumn{2}{|c|}{ QUADRO I. Pedido de ECG de acordo com a idade } \\
\begin{tabular}{l|c} 
Idade & $\%$ Instituições $(n=19)$ \\
\hline Qualquer idade & $26 \%(5)$ \\
\hline$>40 \mathrm{~A}$ & $16 \%(3)$ \\
\hline$>45 \mathrm{~A}$ & $16 \%(3)$ \\
\hline$>50 \mathrm{~A}$ & $32 \%(6)$ \\
\hline$>55 \mathrm{~A}$ & $5 \%(1)$ \\
\hline$>50$ se $\%>65$ se ơ & $5 \%(1)$ \\
\hline
\end{tabular}
\end{tabular}

que poderia atingir $€ 41,66$. Este valor máximo refere-se às situações em que a instituição requere, adicionalmente, radiografia de tórax e espirometria. Verifica-se que numa instituição o custo máximo é difícil de calcular, uma vez que a instituição refere como necessário um estudo detalhado das comorbilidades apresentadas pelos utentes.

Por fim, considerando o número de colonoscopias realizadas no ano de 2015 no ACeS Porto Ocidental (3.072), podemos estimar um gasto entre um valor mí-

\section{QUADRO II. Dados obtidos relativamente aos procedimentos e custos de cada convencionado}

\begin{tabular}{|c|c|c|c|c|c|c|c|c|c|}
\hline Convencionado & $\begin{array}{l}\text { Hemog. } \\
\text { c/ Plaq. }\end{array}$ & $\begin{array}{c}\text { Est. } \\
\text { coagulação }\end{array}$ & Glicose & $\begin{array}{c}\text { Função } \\
\text { renal }\end{array}$ & lonograma & ECG & $\begin{array}{l}\text { Outros exames de } \\
\text { acordo com } \\
\text { comorbilidades }\end{array}$ & $\begin{array}{l}\text { Min. } \\
\in\end{array}$ & $\begin{array}{c}\text { Máx. } \\
\in\end{array}$ \\
\hline A & & $X$ & & & & $x$ & - & \multicolumn{2}{|c|}{10,38} \\
\hline$B, C$ e D & $x$ & $x$ & & & & $*$ & - & 11,51 & 15,38 \\
\hline$E$ & $x$ & $x$ & & & & $*$ & Ecocardiograma & 11,51 & 56,08 \\
\hline $\mathrm{F}$ & $x$ & $x$ & & $x$ & & $*$ & - & 14,11 & 17,98 \\
\hline G & $x$ & $x$ & $x$ & $x$ & & $*$ & - & 15,31 & 19,18 \\
\hline $\mathrm{H}$ & $X$ & $x$ & $X$ & $x$ & & $*$ & $\begin{array}{l}\text { Espirometria } \\
\text { + RX Tórax }\end{array}$ & 15,31 & 41,66 \\
\hline I, J e K & $x$ & $x$ & & & & $x$ & - & \multicolumn{2}{|c|}{15,38} \\
\hline$L, M, N, O$ e $P$ & $x$ & $x$ & $x$ & $x$ & $x$ & $*$ & - & 16,91 & 20,78 \\
\hline Q & $X$ & $x$ & $x$ & $X$ & $x$ & $*$ & Função tiroideia & 16,91 & 31,68 \\
\hline $\mathrm{R}$ & $x$ & $x$ & $x$ & $x$ & $\mathrm{x}$ & * & Estudo «detalhado» & 16,91 & - \\
\hline$S$ & $X$ & $x$ & $X$ & $X$ & $X$ & $X$ & - & 20,78 & \\
\hline
\end{tabular}

Nota: *pedido de ECG de acordo com faixa etária (ver Quadro I).

em presença de patologia pulmonar (doença pulmonar obstrutiva crónica/asma/bronquite), solicitava radiografia de tórax e espirometria; outra requeria relatório médico das comorbilidades; uma instituição pedia função tiroideia perante antecedentes de patologia ou cirurgia tiroideia; outra instituição requeria ecocardiograma face a antecedentes de acidente vascular cerebral ou enfarte agudo do miocárdio; uma instituição referia, se necessário, avaliação prévia feita pela anestesiologia e consequente orientação dos MCDT (Quadro II). Relativamente ao custo dos MCDT verificou-se um valor mínimo de €10,38 e um valor máximo nimo de $€ 31.887,36$ e um valor máximo de $€ 127.979,52$. Deste modo, a variabilidade de procedimentos envolve uma diferença estimada de $€ 96.092,16$.

\section{DISCUSSÃO}

Com base nestes resultados é fundamental que seja feita uma discussão por parte de todos os profissionais envolvidos nestes procedimentos. Consideramos importante o esclarecimento da influência do tipo de sedação na qualidade das colonoscopias efetuadas. Questionamos se esta relação depende na maior parte dos casos da capacidade técnica do gastroenterologista, 
sendo por isso independente da qualidade da sedação. $\mathrm{Ou}$, por outro lado, exames complementares de diagnóstico mais completos significam melhor sedação e, consequentemente, colonoscopias de maior qualidade?

Apesar de não ter feito parte dos objetivos desde estudo constatou-se a frequente utilização, por parte dos prestadores, de nomenclatura não normalizada (análises gerais, bioquímica simples, teste de coagulação) que merece alguma reflexão e até talvez um estudo direcionado à definição destes termos, tendente à sua uniformização na comunicação entre os diversos profissionais. A variabilidade verificada relativamente ao pedido dos ECG reflete uma ausência de uniformização relativa a critérios que justifiquem a realização deste exame.

Nas normas de avaliação e garantia da qualidade da endoscopia digestiva em Portugal vemos esclarecidas as questões relativas à avaliação do doente, estratificação do risco e preparação apenas nos indicadores de qualidade da endoscopia digestiva alta. ${ }^{5} \mathrm{Na}$ literatura internacional, uma revisão de 2013 opta por abordar a endoscopia digestiva na sua globalidade salientando a necessidade da anamnese e exame físicos, especialmente nos casos em que será utilizada uma sedação moderada ou profunda. É considerado um aspeto essencial a existência da doença cardiovascular, respiratória, neurológica, psiquiátrica, endócrina, renal ou de coagulopatias. ${ }^{6}$ Questionamos o porquê da ausência de normas relativas à avaliação do doente e estratificação do risco nos indicadores de qualidade das colonoscopias. Numa realidade em que os utentes apresentam mais comorbilidades existem intervenções técnicas cada vez mais complexas, sendo necessária uma prática custo-efetiva. É, assim, crucial discutir e uniformizar estas normas.

Uma avaliação médica prévia ao procedimento poderá determinar caso a caso o nível de sedação preferível para cada doente, minimizando assim a imprevisibilidade e práticas desnecessárias inerentes ao procedimento. ${ }^{7}$ Qual o papel da história clínica, exame físico, história medicamentosa e classificação ASA na sedação?

Por fim, considera-se relevante a resposta às seguintes questões: as diferentes instituições estão a cumprir os indicadores de qualidade das colonoscopias?
Como e quem está a fazer esta monitorização? Qual a taxa de realização de colonoscopias totais completas das diferentes instituições? Os insucessos na execução do procedimento são justificados? Qual a taxa de complicações de cada instituição e quais as mais frequentes? Estarão os exames requeridos por estas instituições associados a uma melhoria destes fatores?

\section{CONCLUSÕES}

Não sendo este o objetivo do trabalho, e não tendo por isso dados suficientes, não podemos afirmar/concluir qual a instituição com a prática mais adequada.

Na nossa prática somos desafiados a prestar serviços e cuidados de saúde de qualidade custo efetivos, baseados na evidência científica e no respeito por padrões de qualidade definidos por entidades de referência e reconhecidos pelo Ministério da Saúde. Assim, individualmente, por equipas, unidade, serviço e organização, fica clara a necessidade de identificar problemas específicos e requerer respostas adequadas.

Este trabalho identifica uma área suscetível de uniformização com melhoria da eficiência através da elaboração de um documento de consenso que permita diminuir a variabilidade destes procedimentos e garantir que as melhores práticas estão a ser instituídas.

É também identificada uma área com necessidade de uma maior integração de cuidados, da qual resultaria um aumento da satisfação dos utentes e médicos de família.

\section{REFERÊNCIAS BIBLIOGRÁFICAS}

1. Direção-Geral da Saúde. Prescrição de colonoscopia: norma $n^{\circ}$ 004/2014, de 31/03/2014, atualizada em 06/11/2014. Lisboa: DGS; 2014.

2. Gillies RR, Shortell SM, Anderson DA, Mitchell JB, Morgan KL. Conceptualizing and measuring integration: findings from the health systems integration study. Hosp Health Serv Adm. 1993;38(4):467-89.

3. Devers KJ, Shortell SM, Gillies RR, Anderson DA, Mitchell JB, Erickson KL. Implementing organized delivery systems: an integration scorecard. Health Care Manage Rev. 1994;19(3):7-20.

4. Santos I, Sá E. Estratégias de governação clínica [Clinical governance strategy]. Rev Port Clin Ger. 2010;26(6):606-12. Portuguese

5. Sociedade Portuguesa de Endoscopia Digestiva. Normas de avaliação e garantia da qualidade da endoscopia digestiva em Portugal [Internet]. Coimbra: SPED; 2009 [cited 2016 May 17]. Available from: http://www.sped.pt/images/Publicacoes_SPED/SPED_2011052515234 2_Normas_de_Qualidade_em_Endoscopia_Digestiva.pdf

6. Kang $\mathrm{SH}$, Hyun JJ. Preparation and patient evaluation for safe gastrointestinal endoscopy. Clin Endosc. 2013;46(3):212-8. 
7. Vargo JJ, DeLegge MH, Feld AD, Gerstenberger PD, Kwo PY, Lightdale JR, et al. Multisociety sedation curriculum for gastrointestinal endoscopy. Gastrointest Endosc. 2012;76(1):e1-25.

\section{CONFLITO DE INTERESSES}

Os autores declaram não ter quaisquer conflitos de interesse.
ENDEREÇO PARA CORRESPONDÊNCIA

Margarida Vaz Pinto

E-mail: margavazpinto@gmail.com

http://orcid.org/0000-0001-6781-9510

Recebido em 22-06-2016

Aceite para publicação em 03-04-2018

\section{ABSTRACT}

\section{PRESCRIPTION OF ADDITIONAL TESTS TO PERFORM COLONOSCOPY UNDER SEDATION}

In the current context of health resources, efficiency is a crucial goal. This matter is essential and one of the fundamental principles of general practice/family medicine.

Objectives: To characterise the procedures related with the prescription of additional tests to perform colonoscopy with sedation, of the institutions under convention with the North Regional Health Administration.

Methods: The institutions of Porto Ocidental Health Centre Group (ACeS) that provide colonoscopy under sedation to National Health Services (NHS) beneficiaries were contacted, in March 2016, by telephone. The questions focused on the additional exams required to perform colonoscopy under sedation, for how long the additional tests were valid, and on the differences on procedures according to age and presence of co-morbidities.

Results: Twenty-two institutions were contacted, and 19 responses were obtained - response rate of $86 \% ; 100 \%$ of the institutions request international normalized ratio (INR), 95\% haemogram with platelet count; $58 \%$ kidney function; $53 \%$ glucose; $42 \%$ ionogram; and $100 \%$ EKG. There were no standard criteria regarding the prescription of EKG or other specific procedures related with the presence of co-morbidities. The period in which the additional tests were valid varied between three and six months. The total cost of additional tests varied between a minimum of $€ 10.38$ and a maximum of $€ 41.66$. Considering the number of colonoscopies performed in the year 2015 at ACeS Porto (3072), it is estimated that the cost of these tests can vary between $€ 31,887.36$ and $€ 127,979.52$, with an estimated difference in procedures of $€ 96,092.16$.

Discussion: Differences were observed in the type of care provided, in the clarity of the information shared, in the knowledge of the additional exams performed, and in the criteria for request of EKG.

Conclusions: There is a clear need to develop a consensus document that will allow to reduce the variability of these procedures, avoid unnecessary procedures/expenses, ensuring safety and best practice regarding to these procedures.

Keywords: Colonoscopy; Diagnostic tests; Efficiency, Organisational. 